\title{
Studying the unfolding process of protein $G$ and protein $L$ under physical property space
}

\author{
Liling Zhao ${ }^{1,2}$, Jihua Wang ${ }^{* 1,2}$, Xianghua Dou ${ }^{1,2}$ and Zanxia Cao ${ }^{1,2}$
}

Address: ${ }^{1}$ Key Lab of Biophysics in universities of Shandong (Dezhou University), Dezhou 253023, PR China and ${ }^{2}$ Department of Physics, Dezhou University, Dezhou 253023, PR China

Email: Liling Zhao - zhaoll@sina.com; Jihua Wang* - jhwyh@yahoo.com.cn; Xianghua Dou - douxianghua@dzu.edu.cn;

Zanxia Cao - qiayilai@mail.ustc.edu.cn

* Corresponding author

from The Seventh Asia Pacific Bioinformatics Conference (APBC 2009)

Beijing, China. 13-16 January 2009

Published: 30 January 2009

BMC Bioinformatics 2009, I0(Suppl I):S44 doi:I0.II86/I47I-2105-I0-SI-S44

This article is available from: http://www.biomedcentral.com/I47I-2/05/I0/SI/S44

(c) 2009 Zhao et al; licensee BioMed Central Ltd.

This is an open access article distributed under the terms of the Creative Commons Attribution License (http://creativecommons.org/licenses/by/2.0),

which permits unrestricted use, distribution, and reproduction in any medium, provided the original work is properly cited.

\begin{abstract}
Background: The studies on protein folding/unfolding indicate that the native state topology is an important determinant of protein folding mechanism. The folding/unfolding behaviors of proteins which have similar topologies have been studied under Cartesian space and the results indicate that some proteins share the similar folding/unfolding characters.

Results: We construct physical property space with twelve different physical properties. By studying the unfolding process of the protein $G$ and protein $L$ under the property space, we find that the two proteins have the similar unfolding pathways that can be divided into three types and the one which with the umbrella-shape represents the preferred pathway. Moreover, the unfolding simulation time of the two proteins is different and protein $L$ unfolding faster than protein $G$. Additionally, the distributing area of unfolded state ensemble of protein $L$ is larger than that of protein $G$.

Conclusion: Under the physical property space, the protein $G$ and protein $L$ have the similar folding/unfolding behaviors, which agree with the previous results obtained from the studies under Cartesian coordinate space. At the same time, some different unfolding properties can be detected easily, which can not be analyzed under Cartesian coordinate space.
\end{abstract}

\section{Background}

Most proteins exist in unique three-dimensional conformations exquisitely suited to their function. Protein folding is one of the important and unsolved problems in life science. Some sophisticated theories have been proposed after several decades of extensive research through experimental and theoretical studies. The most popular theory is that native state topology is an important determinant of protein folding mechanism $[1,2]$. Studies on some small single-domain proteins suggest that proteins that have similar native structures with low sequence identity have similar transition state ensemble $[3,4]$ and folding rates of two-state proteins have shown to correlate very well with contact order, a quality linked to topology $[5,6]$. On the other hand, there are also some exceptions. Some studies indicated that proteins with the similar native structures maybe have different folding pathway [7-9].

The above studies are usually performed under conformational space or geometrical space that is constructed based on the Cartesian coordinates of atoms. The three-dimensional structure of protein is changed during the folding/ unfolding process. Companied with transformation of three-dimensional Cartesian coordinates of atoms, some physical parameters, such as native contact number, accessible surface area, radius of gyration, are correspondingly 
changed. Some parameters, such as the fraction of the native contacts $\mathrm{Q}[10]$, number of unfolded links $\mu$, and the fraction of residues that are ordered $\mathrm{N}_{f}[11]$, have been chosen as reaction coordinates to depict the protein folding/unfolding process. That is to say, physical property parameters representing some properties of protein can describe the characters during the process of protein folding/unfolding as the atoms three-dimensional Cartesian coordinates do. In this study, we investigate the protein folding/unfolding behaviors under physical property space which is based on physical property parameters. Some novel characters of protein folding/unfolding can be revealed under this physical property space [12].

The B1 IgG binding domain of streptococcal protein G (referred as protein $\mathrm{G}$ in this paper) and the B1 IgG binding domain of peptostreptococcal protein $\mathrm{L}$ (referred as protein $\mathrm{L}$ in this paper) share the similar native state topology. They both consist of a single $\alpha$-helix packed against a four-stranded $\beta$-sheet formed by two symmetrically opposed $\beta$-hairpins (Fig. 1) [13-15] and have low homology (16\% sequence identity). In this study, a physical property space was constructed based on twelve physical property parameters. Protein G and protein L were chosen as model systems to study the unfolding behaviors of proteins with similar native topology under the physical property space. Protein G has been studied by experiments and simulations as a model protein and possessed of many results to be compared. But protein $\mathrm{L}$ has not been studied by simulations as protein $\mathrm{G}$ widely. The results of protein $\mathrm{L}$ could be contrasted with that of protein $G$, as well as its former results. For each protein, forty independent thermal unfolding simulations were performed. With principal component analysis, the multidimensional physical property space had been reduced to three-dimensional essential property subspace. The unfolding trajectories and the unfolded state ensembles of the two proteins were studied under the essential subspace, and some results were different from those obtained under Cartesian coordinate space.

\section{Results \\ Unfolding trajectory}

The unfolding trajectories obtained from the simulations with atoms coordinate of the two proteins were changed into unfolding property trajectories and projected into essential property subspace. The forty unfolding trajectories of each protein could be divided into three types in the subspace (Table 1 ). The trajectory number for type I, II and III were 22, 7, 11 for protein G and 22, 9, 9 for protein $\mathrm{L}$, respectively. For the convenience of observation, only 80 points were selected uniformly from twelve-nanosecond trajectory to draw the figures (Fig. 2 and Fig. 3). One point represented one snapshot of protein during the simulations.
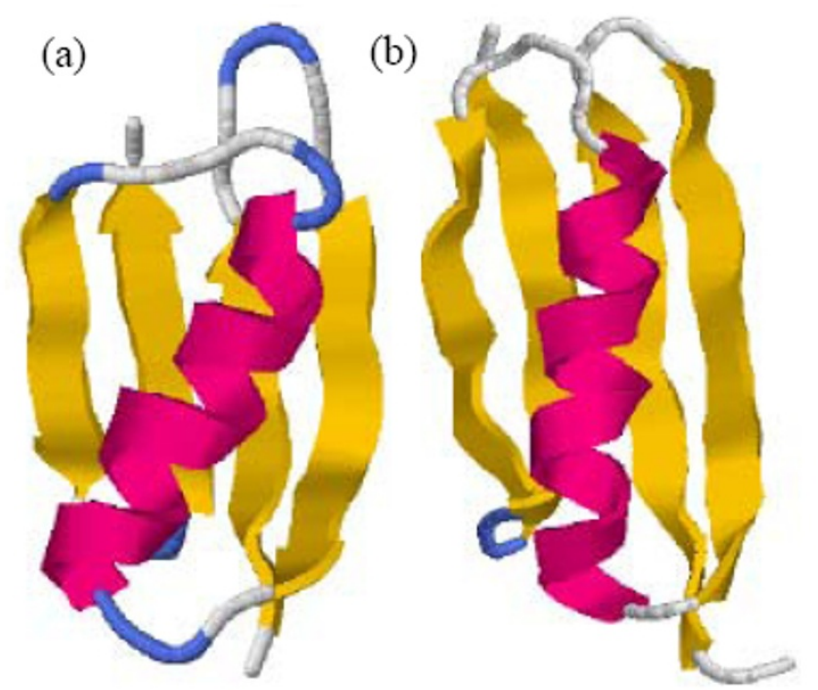

Figure I

The structure of (a) protein G ( $\underline{2 G B I}$ in PDB) and (b) protein $L(\underline{2 P T L}$ in PDB).

Type I had the same shape as umbrella (see Fig. 2(a) and Fig. 3(a)), and this type had 22 (55\%) trajectories among forty unfolding trajectories for the two proteins. The probability of this type was much higher than that of the others.

\section{Unfolded state ensemble}

When the native contact number in a conformation is less than $20 \%$ of that in the native state, the conformation was defined to be in the unfolded state ensemble [16]. Depended on the definition, fifty-five native contacts formed by fifty-six residues were identified for protein $G$, and forty-nine native contacts formed by sixty-two residues were identified for protein $\mathrm{L}$.

The Unfolded states in each trajectory were mapped into the essential property subspace. The forty trajectories had the similar unfolded state ensemble under the subspace for two proteins (Fig. 4). All the unfolded state assembles had the similar ellipsoid shape. The change ranges of the three principal components for unfolded state ensembles of two proteins were calculated (Table 2). The distributing area of unfolded state ensemble of protein L under the subspace was larger than that of protein $\mathrm{G}$.

Table I: The trajectory number of different unfolding types

\begin{tabular}{cccc}
\hline Protein & type I & type II & type III \\
\hline Protein G & 22 & 7 & II \\
Protein L & 22 & 9 & 9
\end{tabular}


(a)

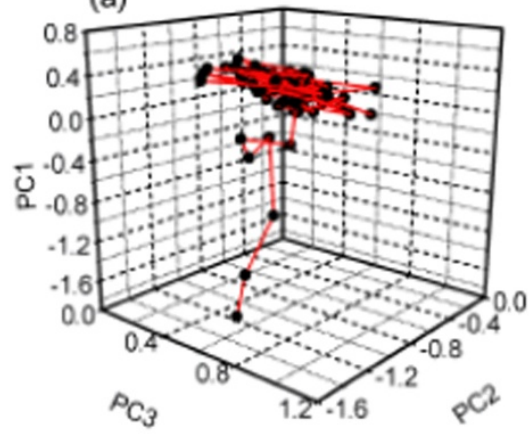

(b)

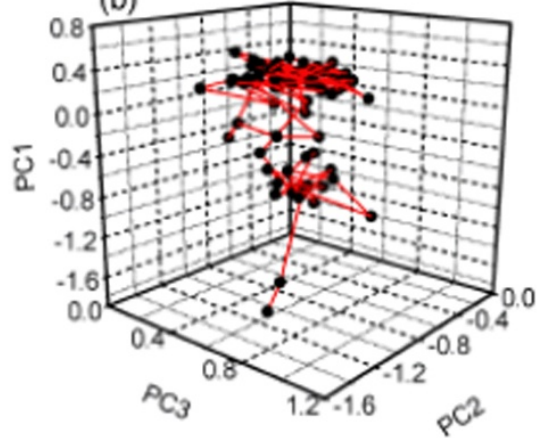

(c)

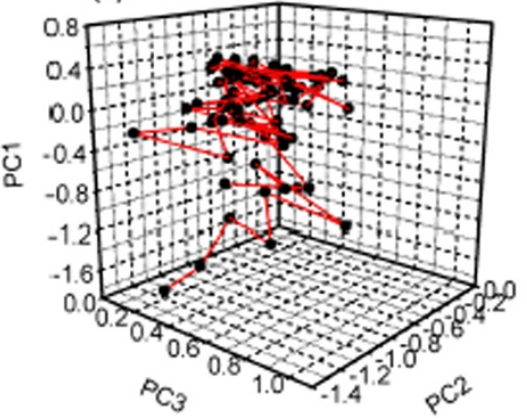

Figure 2

The thermal unfolding trajectories of protein $\mathbf{G}$ under essential property subspace. Eighty points were selected uniformly from twelve-nanosecond unfolded property trajectory to draw the figure. The forty unfolded property trajectories can be divided into three types; they were (a) type I, (b) type II and (c) type III, represented by trajectory 28 , trajectory I2 and trajectory 29 , respectively.

\section{Unfolding simulation time}

The time of the simulation before the protein unfolded into the unfolded state ensemble was called unfolding simulation time. The unfolding simulation time was employed to describe approximately unfolding rate for it is not the actual protein unfolding time. The actual unfolding time must be measured by experiment. The average unfolding simulation time of each unfolding type was calculated (Table 3). The average unfolding simulation time of protein $L$ was approximately two thirds of that of protein $G$, but to type II, protein $G$ unfolded approximately as faster as protein L. For protein $G$, the type II unfolded slower than type I and faster than type III. However, for protein L, the type II unfolded slower than type I and type III. Among the three unfolding types, type I unfolded fastest for both proteins.

\section{Discussion}

One parameter can describe one property of protein. The more parameters were selected, the better conformation of the protein was represented. We selected twelve physical parameters [see Methods], which were often used in (a)

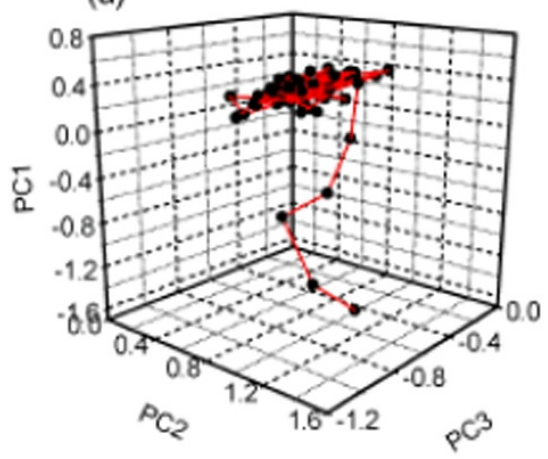

(b)

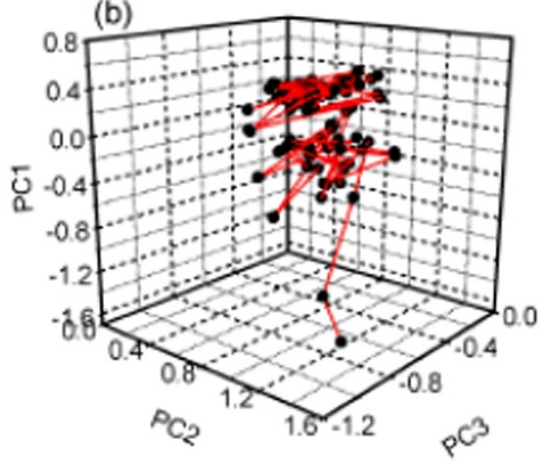

(c)

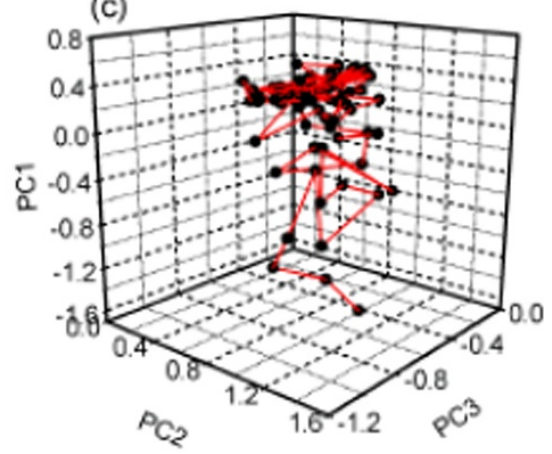

Figure 3

The thermal unfolding trajectories of protein $L$ under essential property subspace. Eighty points were selected uniformly from twelve-nanosecond unfolded property trajectory to draw the figure. The forty unfolded property trajectories can be divided into three types; they were (a) type I, (b) type II and (c) type III, represented by trajectory I7, trajectory I4 and trajectory 35 , respectively. 

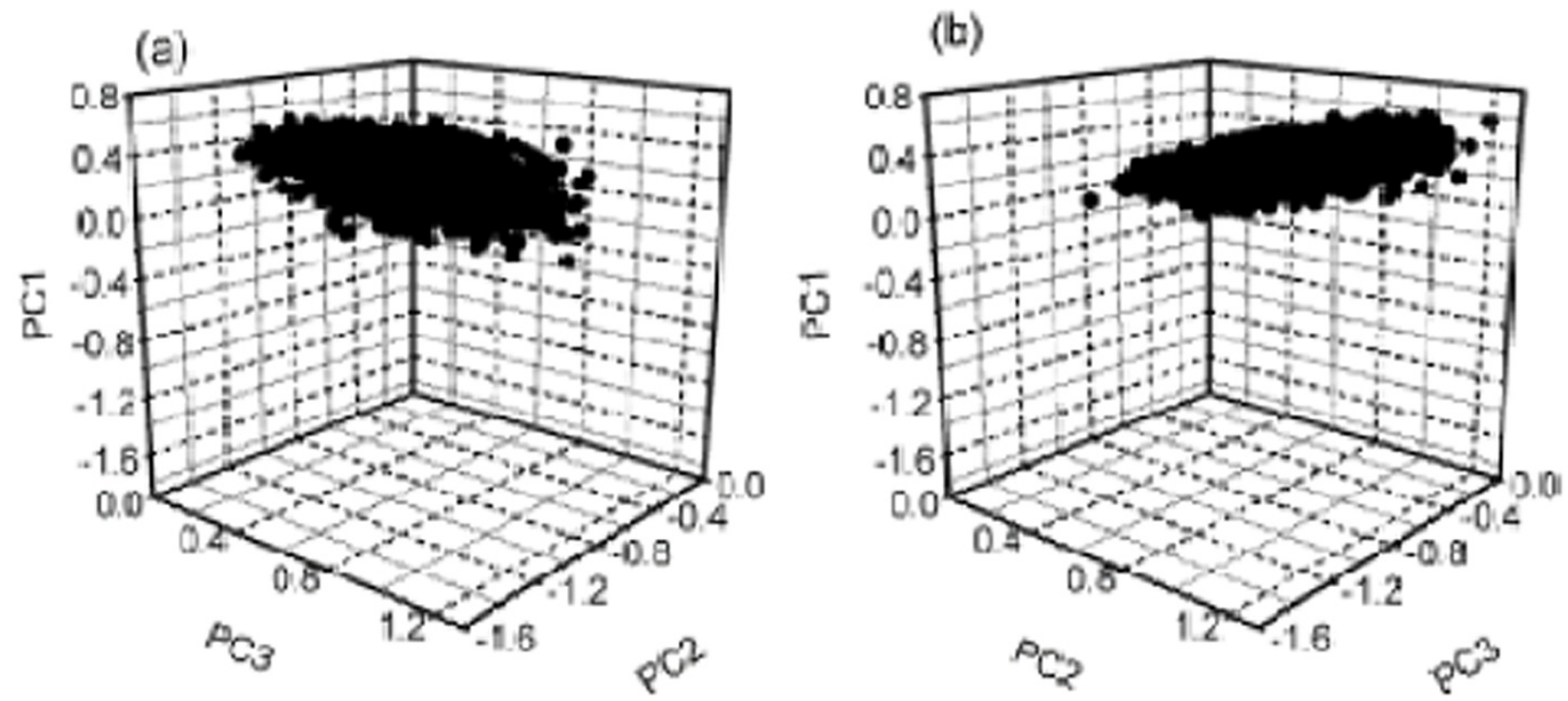

\section{Figure 4}

The unfolded state ensemble of protein $\mathbf{G}$ and protein $\mathbf{L}$ under essential property subspace. The unfolded state ensemble have the ellipsoid shape for (a) protein $\mathrm{G}$ and (b) protein L, represented typically by trajectory 28 and trajectory I7, respectively.

protein folding/unfolding analysis, to depict the character of protein at each time-step during the unfolding process. It was the changes of protein structure that were studied under Cartesian coordinate space. However, it was the changes of protein properties that were studied under physical property space. We could analyse the transformation of protein properties during the folding/unfolding process. Decreasing the twelve-dimension space to threedimensional essential subspace could make the property trajectory be observed easily. The top three eigenvalues were much higher than the others [Fig. 5], which ensured that the subspace covered the main characters of twelvedimension space. The different physical parameters has different loading for the three principal components for two proteins [Fig. 6]

With the protein spread, the native contact number $\mathrm{N}$ decreased, at same time, the total contact distance TCD became smaller and the second structure content SSC including the $\beta$ sheet content decreased. For the inverse proportion with those parameters, the first principal component PC1 increased accordingly (Fig. 2, 3). The second principal component PC2 was dominated by the loading of radius of gyration of $\mathrm{C}_{\alpha}$ atom $\mathrm{Rg} 1$ and number of hydrogen bonds between the protein and water HB4. During the unfolding process, the protein unwound and its volume became bigger, and the more hydrogen bonds between the protein and water were formed, then the second principal component PC2 was increased in physical property space until the unfolding simulation convergence. The variety of PC3 was the same as PC1 and PC2.

Under the essential property subspace, the unfolding trajectories of protein $\mathrm{G}$ and protein $\mathrm{L}$ had the similar three types, which was coordinate with the fact that native state topology determines the folding mechanism. Type I with umbrella-shape had the higher appearance probability and the shortest unfolded simulation time among the three types, which might induced that this type was the preferred pathway among the multiple pathway [17]. The shape of unfolding trajectory of type I indicated that the

Table 2: The three principal components of unfolded states ensemble

\begin{tabular}{cccccc}
\hline Protein & Component & $\max$ & aver & $\min$ & range \\
\hline \multirow{2}{*}{ Protein G } & PCI & 1.125 & 0.438 & -0.127 & 1.252 \\
& PC2 & 0.049 & -0.662 & -1.517 & 1.565 \\
& PC3 & 0.735 & 0.250 & -0.386 & 1.121 \\
Protein L & PCI & 0.240 & -0.406 & -1.146 & 1.386 \\
& PC2 & 1.478 & 0.606 & -0.145 & 1.622 \\
& PC3 & 0.785 & 0.245 & -0.491 & 1.276 \\
\hline
\end{tabular}


Table 3: The average unfolding simulation time of each unfolding type (ps)

\begin{tabular}{ccccc}
\hline Protein & whole & type I & type II & type III \\
\hline Protein G & 2822 & 2064 & 3345 & 4004 \\
Protein L & 2134 & 1367 & 3391 & 2763 \\
\hline
\end{tabular}

two proteins were fast folding two-state proteins, which was consistent with the fact that protein $\mathrm{G}$ and protein $\mathrm{L}$ are fast folding two-state kinetics proteins [18,19]. For the similar topology, protein $\mathrm{G}$ and protein $\mathrm{L}$ had the similar unfolded state ensemble as ellipsoid.

Protein G and protein L have low homology (16\% sequence identity) although they share the similar native state topology. The most obvious difference for the two proteins is the $\alpha$-helix orientation. In protein $L$ the helix is almost parallel to the $\beta$-sheet, whereas in the protein $G$ the helix runs diagonally across the sheet [15]. Studies indicate that one of the two $\beta$-turns is largely formed and the other largely disrupted in the folding transition state. In protein $\mathrm{L}$ it is the $\mathrm{N}$-terminal $\beta$-turn, and in the protein $\mathrm{G}$ the $\mathrm{C}$-terminal $\beta$-turn, that is formed in the transition ensemble $[7-9,20]$. However, the difference disappeared under the property space for the two proteins. The property space was constructed by physical property parameters of protein, for the two proteins, different transient states in Cartesian space might have the same physical properties. At same time, some other difference of unfolding behaviors of the two proteins was observed obviously under the property space.

First, the unfolding simulation time was difference. Protein L unfolded much faster than protein $G$, especially for the type I which represented the preferred pathway, which was accordant with the experiments study $[19,21]$. It may be related to the amino acid sequence of the two proteins. Protein $\mathrm{G}$ and protein $\mathrm{L}$ had different residue number and different native contact number though they had the similar native topology structures. The ratio of local contact among all native contacts was different between protein $G$ and protein $L[7,8]$ and the local and non-local contacts had different influence on unfolding rate.

Second, the distributing area of unfolded state ensemble was different. The unfolded states resided in a finite area under the property space, which agreed with the fact that the unfolded states are not infinite but finite [22,23]. For protein $\mathrm{L}$, the change ranges of three principal components were larger than those of protein $G$, and corresponding distributing area of unfolded state ensemble of protein L was larger than that of protein G. With similar native topology, protein $\mathrm{L}$ had the more unfolded states.
Among the multiple folding pathways, the unfolding difficulty of protein was different. Protein L had more unfolding difficulty by type II than by the other two types and protein $\mathrm{G}$ had middle unfolding difficulty by type II among the three unfolding types. For the same reason, protein L unfolded slower than protein G by type II.

The closer of the two points were in property subspace, the more similar properties the two conformations had. For type II, proteins unfolded from native state to unfolded state with an obvious stagnation at some states. There might be a intermediate state for the two proteins, which required further study to be confirmed.

In the physical property space, a point represents a conformation of protein that have thousands of atom coordinates in Cartesian space. The whole behaviors of protein folding/unfolding can be observed easily. With some effective analysis tool such as network, some details of protein folding mechanism may be detected, which is worthy of the following study.

\section{Conclusion}

In this study, the physical property space was constructed with twelve physical parameters and decreased to threedimensional essential property subspace. Under the property space, the unfolding behaviors of protein $\mathrm{G}$ and protein $\mathrm{L}$ were studied. With the statistical analysis on the forty unfolding property trajectories, we found that the two proteins with similar native state topologies had the similar unfolding property trajectories and similar unfolded state ensemble under the property space, which agreed with the previous study under Cartesian space. At the same time, some unfolding properties, which could not be realized by studies under Cartesian space, could be easily detected, for example, the unfolding pathway type,

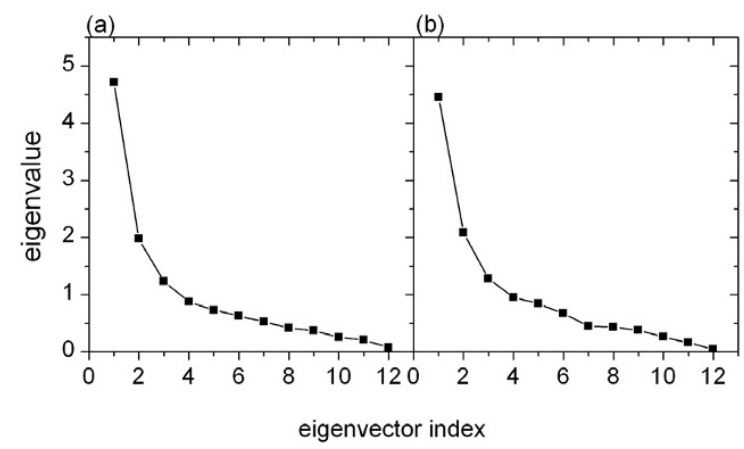

Figure 5

The twelve eigenvalues of (a) protein $\mathbf{G}$ and (b) protein $\mathbf{L}$. The eigenvalues were obtained from average property covariance matrix in decreasing order of magnitude. 

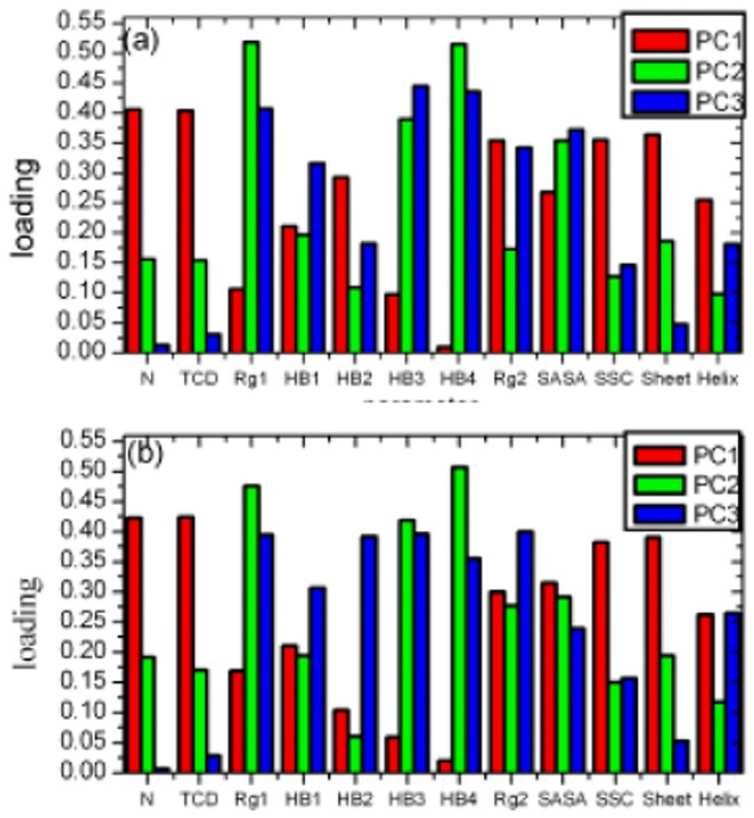

parameter

\section{Figure 6}

The loadings of physical parameters for first three principal components of two proteins. (a) protein $\mathrm{G}$, (b) protein $L . N$ is native contact number within the protein; TCD is total contact distance; $\mathrm{Rgl}$ is radius of gyration of $\mathrm{C}_{\alpha}$ atom; $\mathrm{HBI}$ is number of hydrogen bonds between the protein and waters within the hydrophobic core, HB2 is number of hydrogen bonds within the hydrophobic core, HB3 is number of hydrogen bonds within the protein, HB4 is number of hydrogen bonds between the protein and water, $\mathrm{Rg} 2$ is radius of gyration of the hydrophobic core, SASA is hydrophobic solvent-accessible surface area, SSC is second structure content, Sheet is the content of $\beta$ sheet, Helix is the content of $\alpha$ helix.

the difference of unfolding simulation time and the difference of distributing area of the unfolded state ensemble. At last, we only studied the two proteins and can not say that all proteins with similar native topology have the same characteristic under property space as protein $\mathrm{G}$ and protein $\mathrm{L}$, which demands more deep research.

\section{Methods}

\section{Molecular dynamics (MD) simulations}

The initial conformations of protein $\mathrm{G}$ and protein $\mathrm{L}$ were taken from protein data bank (PDB) [24] with PDB entry code 2GB1 and 2PTL, respectively, which have been solved by NMR spectroscopy [13-15]. Unfolding simulations were carried out using the GROMACS software package [25] with the GROMOS96 43a1 force field [26] and explicit water. The SPC water model was used for water molecules [27]. After energy minimization, some water molecules were replaced with same number of chlorine or natrium ions to neutralize the system. Under $300 \mathrm{~K}$ and 1 bar, position-restrained MD simulations were performed for $500 \mathrm{ps}$ and forty conformations were received every 10 ps from the last 400 ps simulation trajectory. With each conformation, position-restrained MD simulations were performed for 100 ps under $540 \mathrm{~K}$ and 1 bar at first, and then free MD simulations were carried out for $12 \mathrm{~ns}$ under same condition. The time step of simulation is $2 \mathrm{fs}$ and the total simulation time is up to $0.96 \mu \mathrm{s}$.

\section{Physical property}

For the convenience of analysis, 6000 conformations were chosen for the following analysis from 12 ns unfolding trajectories with same interval. The following twelve physical properties were calculated, they are $\alpha$ helix content, $\beta$ sheet content, second structure content (including $\alpha$ helix, $\beta$ sheet, $\beta$ bridge, bend and turn), hydrophobic solventaccessible surface area, radius of gyration of $\mathrm{C}_{\alpha}$ atom, number of hydrogen bond within the protein, radius of gyration of the hydrophobic core, number of hydrogen bond within the hydrophobic core, number of hydrogen bond between the protein and water, number of hydrogen bond between the protein and waters within the hydrophobic core, native contact number within the protein, and total contact distance (TCD) [20].

We define a contact as being present if the $\mathrm{C}_{\alpha}$ atom of two residues $(i, j)$ are within 6.5 angstrom. We define native contact including all contact formed between residues not adjacent in sequence and present in both reference native simulations for more than two-thirds of the simulation time [16].

\section{Principal component analysis and property space}

The twelve parameters mentioned above of protein were calculated for each conformation during the simulations. The value of each parameter was normalized between 0 and 1 , with 0 corresponding to the lowest value across the trajectory and 1 being the highest value across the trajectory. The covariance property matrix $C$ was calculated and the element $c_{i j}$ was determined by

$$
c_{i j}=\left\langle\left(x_{i}-\left\langle x_{i}\right\rangle\right)\left(x_{j}-\left\langle x_{j}\right)\right\rangle\right.
$$

where $\langle$ donated the average over all structures sampled in the trajectory and $x_{i}=x_{i}(t)$ was the $i^{\text {th }}$ physical parameter of the conformation at time $t$.

The property matrix $C$ was $12 \times 12$ dimensional symmetric matrix. An average property matrix was obtained by averaging forty property matrixes. With the principal component analysis, the average property matrix was diagonalized to get the twelve new orthogonal eigenvectors and 
corresponding eigenvalues. The first three eigenvectors with largest eigenvalues were selected as three principal components $\overrightarrow{P C_{1}}, \overrightarrow{P C_{2}}, \overrightarrow{P C_{3}}$ to construct three-dimensional essential physical property subspace, and the unfolding trajectories were projected into the subspace.

\section{Competing interests}

The authors declare that they have no competing interests.

\section{Authors' contributions}

Liling Zhao carried out most of the theoretical analysis and drafted the manuscript, while Jihua Wang carried out some of the theoretical analyses and designed the research project and Zanxia Cao revised the paper. Xianghua Dou and Liling Zhao performed the molecular dynamics simulations. All authors read and approved the final manuscript.

\section{Acknowledgements}

We gratefully thank Prof. H.J.C. Berendsen for providing us with the GROMACS programs. This work was supported by a grant from Chinese National Key Fundamental Research Project (No. 90403 I 20) and Shandong Fundamental Research Project (NO.Y2005D I2) and project of shandong domestic visitorial researcher for excellent young teacher of universities.

This article has been published as part of BMC Bioinformatics Volume 10 Supplement I, 2009: Proceedings of The Seventh Asia Pacific Bioinformatics Conference (APBC) 2009. The full contents of the supplement are available online at http://www.biomedcentral.com//47/-2105/I0?issue=SI

\section{References}

I. Baker D: A surprising simplicity to protein folding. Nature 2000, 405:39-42.

2. Alm E, Baker D: Matching theory and experiment in protein folding. Curr Opin Struct Biol 1999, 9:189-196.

3. Riddle DS, Grantcharova VA, Santiago JD, Alm E, Ruczinski I, Baker $D$ : Experiment and theory highlight role of native state topology in SH3 folding. Nature Struct Biol 1999, 6:1016-1024.

4. Martinez JC, Serrano L: The folding transition state between $\mathrm{SH} 3$ domains is conformationally restricted and evolutionarily conserved. Nature Struct Biol 1999, 6:1010-1016.

5. Plaxco KW, Simons KT, Baker D: Contact order, transition state placement and the refolding rates of single domain proteins. J Mol Biol 1998, 277:985-994.

6. Ivankov DN, Garbuzynskiy SO, Alm E, Plaxco KW, Baker D, Finkelstein AV: Contact order revisited: influence of protein size on the folding rate. Protein Sci 2003, I 2(9):2057-62.

7. McCallister EL, Alm E, Baker D: Critical role of $\beta$-hairpin formation in protein G folding. Nat Struct Biol 2000, 7:669-673.

8. Karanicolas J, Brooks CLIII: The origins of asymmetry in the folding transition states of protein $\mathbf{L}$ and protein $\mathbf{G}$. Protein $\mathrm{Sci}$ 2002, II:235I-236I.

9. Kim DE, Fisher C, Baker D: A breakdown of symmetry in the folding transition state of protein L. J Mol Biol 2000, 298:97|-984.

10. Cecilla C, Nelson O: Topological and energetic factors: what determines the structural details of the transition state ensemble and "on-route" intermediates for protein folding? An investigation for small globular proteins. I Mol Biol 2000, 298:937-953.

II. Alm E, Baker D: Prediction of protein-folding mechanism from free-energy landscape derived from native structures. PNAS 1999, 96:11305-II310.

12. Ji-Hua W, Li-Ling Z, Xiang-Hua D: Study of Multiple Unfolding Trajectories and Unfolded States of the Protein GB I Under the Physical Property Space. J Biomol Struct Dyn 2008, 25:609-620.

13. Gronenborn AM, Filpula DR, Essig NZ, Achari A, Whitlow M, Wingfield PT, Clore GM: A novel, highly stable fold of the immunoglobulin binding domain of streptococcal protein $\mathbf{G}$. Science 1991, 253:657-66I.

14. Wikstrom M, Sjobring U, Kastern W, Bjorck L, Drakenberg T, Forsen $\mathrm{S}$ : Proton nuclear magnetic resonance sequential assignments and secondary structure of an immunoglobulin light chain-binding domain of protein L. Biochemistry 1993, 32:338I-3386.

15. Wikstrom M, Drakenberg T, Forsen S, Sjobring U, Bjorck L: Threedimensional solution structure of an immunoglobulin light chain-binding domain of protein L. Comparison with the IgG-binding domains of protein G. Biochemistry 1994, 33:14011-14017.

16. Sheinerman FB, Brooks CL: Calculations on folding of segment B I of streptococcal protein G. J Mol Biol 1998, 278:439-456.

17. Lazaridis T, Karplus M: "New view" of protein folding reconciled with the old through multiple unfolding simulations. Science 1997, 278:1928-1931.

18. Alexander P, Orban J, Bryan P: Kinetic analysis of folding and unfolding the $\mathbf{5 6}$ amino acid IgG-binding domain of streptococcal protein G. Biochemistry 1992, 3 I:7243-7248.

19. Scalley ML, Yi Q, Gu H, McCormack A, Yates JR, Baker D: Kinetics of folding of the IgG binding domain of peptostreptococcal protein L. Biochemistry 1997, 36(I I):3373-3382.

20. Hongyi Z, Yaoqi Z: Folding rate prediction using total contact distance. Biophys / 2002, 82:458-463.

21. Plaxco KW, Spitzfaden C, Campbell ID, Dobson CM: A comparison of the folding kinetics and thermodynamics of two homologous fibronectin type III modules. J Mol Biol 1997, 270:763-770.

22. Daura X, Jaun B, Seebach D, van Gunsteren WF, Mark AE: Reversible peptide folding in solution by molecular dynamics simulation. J Mol Biol 1998, 280:925-932.

23. Berman HM, Westbrook J, Feng Z, Gilliland G, Bhat TN, Weissig H, Shindyalov IN, Bourne PE: The Protein Data Bank. Nucleic Acids Res 2000, 28:235-242.

24. Daura X, van Gunsteren WF, Mark AE: Folding-unfolding thermodynamics of a $\beta$-heptapeptide from equilibrium simulations. Proteins Struct Funct Genet 1999, 34:269-280.

25. Scott WRP, Huenenberger PH, Tironi IG, Mark AE, Billete SR, Fennen J, Torda AE, Huber T, Krueger P, van Gunsteren WF: The GROMOS biomolecular simulation program. J Phys Chem A 1999, 103:3596-3607.

26. van Gunsteren WF, Billeter SR, Eising AA, Hünenberger PH, Krüger P, Mark AE, Scott WRP, Tironi IG: The GROMOS96 Manual and User Guide. Vdf Hochschulverlag AG, Zürich; 1996.

27. Berendsen HJC, Grigera JR, Straatsma TP: The missing term in effective pair potentials. J Phys Chem 1987, 91:6269-6271.

Publish with Bio Med Central and every scientist can read your work free of charge

"BioMed Central will be the most significant development for disseminating the results of biomedical research in our lifetime. "

Sir Paul Nurse, Cancer Research UK

Your research papers will be:

- available free of charge to the entire biomedical community

- peer reviewed and published immediately upon acceptance

- cited in PubMed and archived on PubMed Central

- yours - you keep the copyright 